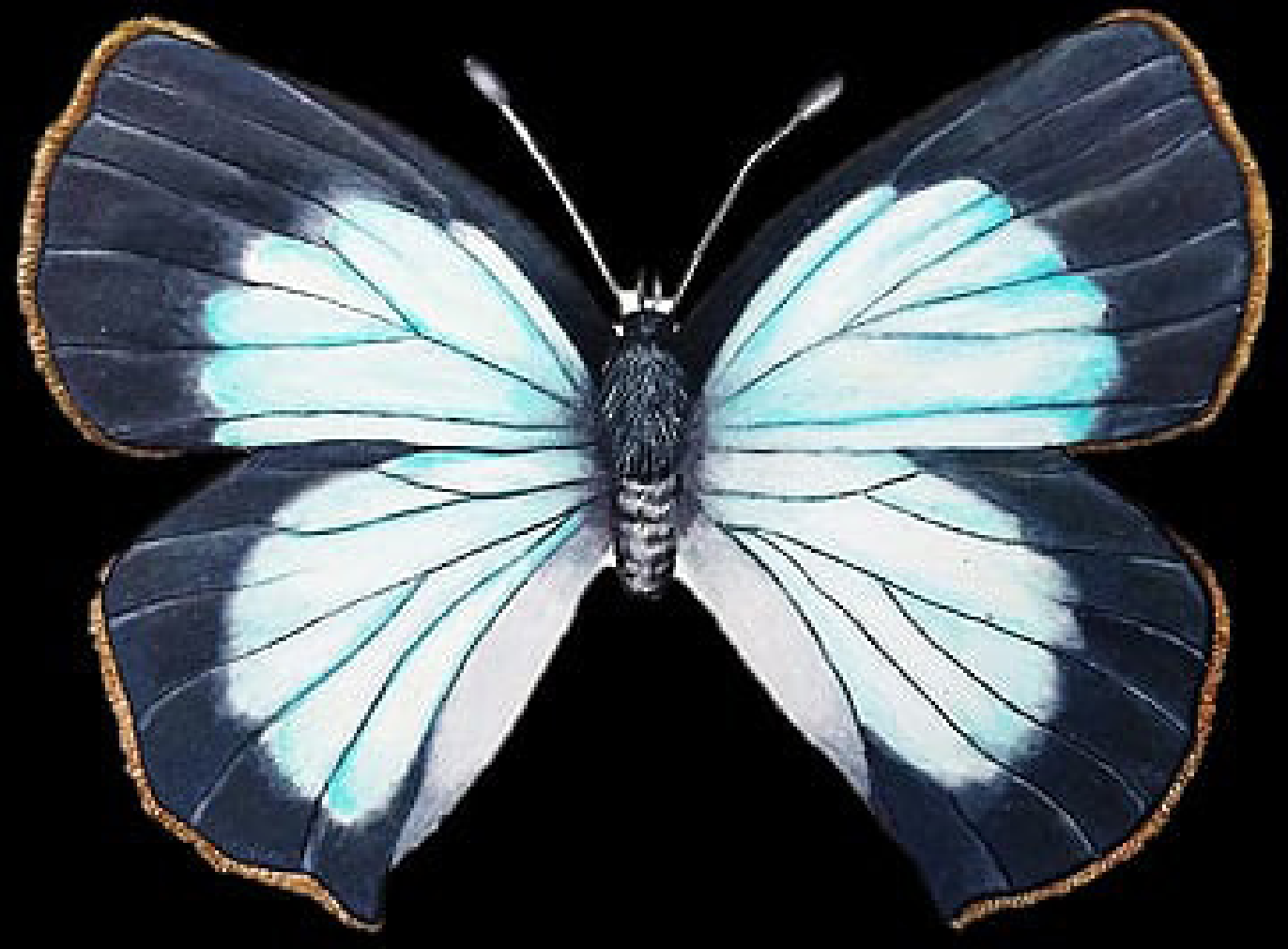

Ilustración: Jeny Fuentes 


\section{SABERES ACERCA DE LA BIODIVERSIDAD EN UN ESCENARIO DE EDUCACIÓN NO CONVENCIONAL}

\section{Knowledge about Biodiversity in an Unconventional Education Scenario}

\section{Conhecimento sobre biodiversidade em um cenário de educação não convencional}

\section{Ludvison Miguel Herrera Urrego*}

\section{Resumen}

El presente artículo de investigación se construye a partir de la experiencia y los resultados de la práctica pedagógica desarrollada durante el 2016 en un escenario de educación no convencional: El Jardín Botánico José Celestino Mutis, en Bogotá. La práctica tuvo como objetivo principal el análisis de los saberes en relación con la biodiversidad en un grupo de estudiantes participantes del Servicio Social Ambiental, mediante la construcción e implementación de una estrategia pedagógica fundamentada en temas relacionados con la biodiversidad. Se siguió una metodología interpretativa, de acuerdo con los planteamientos de Ramírez (2012), con un enfoque cualitativo (González Monteagudo, 2001). El estudio está estructurado en tres fases. Una fase exploratoria, que implica el acercamiento del investigador al contexto y la población; una fase analítica, que considera la caracterización de los saberes en torno a la biodiversidad; y finalmente una fase propositiva, que pone en escena la estrategia pedagógica. Entre las conclusiones se destaca una conceptualización polisémica de la biodiversidad, centrada en la variabilidad de formas de vida, con implicaciones políticas, económicas, sociales y educativas. Respecto a los escenarios no convencionales, se insta a cuestionarse por sus posibilidades y la configuración de nuevos marcos de análisis y participación para los maestros de biología.

Palabras clave: diversidad biológica; estrategia pedagógica; saberes; educación no convencional

* Universidad Pedagógica Nacional. Correo electrónico: Imiguelherrerau1@gmail.com 


\section{Abstract}

This paper is the product of a pedagogical practice developed in 2016 in a non-conventional education setting: the José Celestino Mutis Botanical Garden in Bogotá. The main purpose of the practice was to analyze the knowledge about biodiversity in a group of students engaged in the Environmental Social Service, through the design and implementation of a pedagogical strategy. This three-stage study followed an interpretative methodology, as stated by Ramírez (2012), using a qualitative approach (González Monteagudo, 2001). In the exploratory stage, the researcher approached the context and the population. In the analytical stage, he introduced the students' knowledge about biodiversity. Finally, in the propositive stage, he applied the pedagogical strategy. One of the main results was the polysemic conceptualization of biodiversity, centered on the variability of life-forms. This concept has an impact on the political, economic, social, and educational fields. On the other hand, the author encourages readers to reflect upon the possibilities to turn to non-conventional settings, and think about new analysis and participation contexts for biology teachers.

Keywords: biodiversity; teaching strategy; knowledge; non-conventional education

\section{Resumo}

Este artigo de pesquisa baseia-se na experiência e nos resultados da prática pedagógica desenvolvida ao longo de 2016 em um cenário de educação não convencional: o Jardim Botânico José Celestino Mutis, em Bogotá. A prática teve como objetivo principal a análise do conhecimento em relação à biodiversidade em um grupo de estudantes participantes do Serviço Social Ambiental, através da construção e implementação de uma estratégia pedagógica baseada em questões relacionadas à biodiversidade. Foi adotada uma metodologia interpretativa, de acordo com Ramírez (2012), com abordagem qualitativa (González Monteagudo, 2001). O estudo está estruturado em três fases. Uma fase exploratória, que implica a abordagem do pesquisador quanto ao contexto e à população; uma fase analítica, que considera a caracterização do conhecimento sobre biodiversidade; e, finalmente, uma fase proativa, que coloca a estratégia pedagógica em cena. Entre as conclusões, destaca-se uma conceitualização polissêmica da biodiversidade, com foco na variabilidade das formas de vida, com implicações políticas, econômicas, sociais e educacionais. Em

relação aos cenários não convencionais, recomenda-se questionar suas possibilidades e a configuração de novas estruturas de análise e participação para professores de biologia.

Palavras chave: diversidade biológica; estratégia pedagógica; conhecimento; educação não convenciona 


\section{Introducción}

La práctica pedagógica propuesta en el programa de Licenciatura en Biología de la Universidad Pedagógica Nacional ha establecido un espacio centrado en la interacción, innovación e investigación del futuro maestro de biología (UPN, 2013). Precisamente como resultado de la aplicación de esta normatividad surge el presente texto, que representa el conjunto de acciones que nacen cuando el futuro profesional se sitúa en espacios no ligados al aula tradicional.

En esta investigación seguimos la definición propuesta por Zuluaga (1999), según la cual "El saber es el espacio más amplio y abierto de un conocimiento, espacio donde se localizan discursos de muy diferentes niveles" (p. 26). Desde esta perspectiva es posible indagar y visibilizar los elementos y las interacciones en conjunto, reconociendo su procedencia, sus métodos y las dinámicas que generan. Esto conduce a una reflexión ampliada y a una mirada que permite conocer lo que circula.

En la formación de licenciados de biología es importante destacar la trascendencia de una serie de saberes asociados a la disciplina científica en concreto. La biología, como campo de conocimiento, se centra en el estudio de lo vivo, que construye figuras que reflejan su interés conceptual, por ejemplo, la biodiversidad. A grandes rasgos, este término se refiere a la variedad de formas de vida existentes; no obstante, merece atención en cuanto a los perfiles que la han configurado.

Vale la pena destacar el papel que desempeña la biodiversidad en el contexto actual, y cómo es preciso analizar la emergencia de su discurso desde una perspectiva detallada, que exponga las vertientes en las que tiene lugar. Esto lleva a preguntarse acerca de su valor en el escenario educativo; con tal propósito, este ejercicio surge de una mirada sumamente singular: los escenarios de educación no convencionales. De acuerdo con Trujillo y Valbuena (2015), estos escenarios son potenciales contextos de educación de la sociedad, caracterizados por contenidos y finalidades diversas, que confluyen como una modalidad distinta a los tipos de educación formal, no formal e informal. Así, renuevan los modos para el acercamiento, el reconocimiento y la conceptualización de la biodiversidad.

En este sentido, el reto de explorar las potencialidades de la biodiversidad y su relación con la enseñanza de la biología en un espacio en interacción directa con la variabilidad de lo vivo constituye un insumo para la construcción del conocimiento y una mirada posible desde la pedagogía y el saber pedagógico. En consecuencia, es pertinente situar esta clase de ejercicios en una perspectiva investigativa pedagógica que permita observar los procesos escolares en simultaneidad con la práctica del maestro, para desarrollar así una propuesta crítica y en constante evaluación, en la cual el docente se asume como investigador.

Este escrito se centra en el análisis de los saberes asociados a la biodiversidad, recreados en un escenario educativo no convencional, llamando la atención sobre la construcción de elementos y estrategias. Para ello, se describe la construcción e implementación de una estrategia pedagógica desarrollada en el Jardín Botánico de Bogotá, José Celestino Mutis (JBB), en el marco de las posibilidades del Servicio Social Ambiental (SSA).

\section{¿Qué involucra pensar la investigación en la práctica del maestro?}

La investigación pedagógica es un espacio para la reflexión de quienes tratan con la enseñanza; es un elemento excepcional de los maestros, quienes cuestionan, escriben y reportan el saber escolar, desde el cual surgen preguntas relacionadas con qué, cómo, dónde y para qué se enseña (Calvo, Camargo y Pineda, 2012). En este sentido es el maestro quien se encuentra ante el interrogante por su práctica. Esta mirada le permite experimentar, desde la cotidianidad de su labor, constituyendo un sinnúmero de opciones, tantas como escenarios en donde participa.

A pesar de tal intención, vale traer en consideración la diferencia entre el mencionado concepto y la investigación educativa. De acuerdo con Amaya (2007): "Investigar en educación es entre otras cosas, analizar con rigurosidad y objetividad una situación educativa entendida en sentido amplio" (p. 17). A partir de allí, hablar de investigación educativa implica considerar el escenario educativo en un contexto generalizado, lo que supone la participación no solo de la pedagogía, sino de otras disciplinas que tienen cabida en las llamadas ciencias de la educación. Así las cosas, el desarrollo de la investigación educativa no emana exclusivamente del maestro; también entran en juego elementos provenientes de la sociología, la psicología, la antropología y otras humanidades (Calvo et ál., 2012).

En consecuencia, la investigación pedagógica reconoce el campo del conocimiento que la origina, sus propuestas, discusiones e inferencias, que se deben diferenciar de las de la investigación educativa, que también procede y actúa desde una lógica particular. Así, la investigación pedagógica permite interiorizar la investigación desde la reflexión en particular de los maestros, quienes se interrogan alrededor de su labor y sus posibilidades en el marco

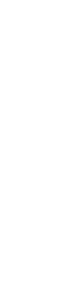


del saber pedagógico al ser artífices del ejercicio de enseñanza. Vale la pena aclarar que la discusión entre la investigación educativa y la investigación pedagógica requiere de un análisis en profundidad, el cual no es objeto de la presente propuesta. Por el contrario, los anteriores párrafos pretenden presentar únicamente la mirada con la que se procede en este análisis.

\section{Pensar la diversidad en la biodiversidad}

Conocer la biodiversidad involucra relacionarse con la diversidad biológica. La mirada más aceptada de este término surge del Convenio sobre la Diversidad Biológica (CDB), en el que se comprende por diversidad biológica la variabilidad de organismos vivos de cualquier fuente. El concepto abarca la diversidad dentro de cada especie, entre las especies, los ecosistemas y los complejos ecológicos de los que forman parte (Organización de las Naciones Unidas, 1992). Desde este enfoque, la diversidad biológica se entiende como la variabilidad existente en los organismos en los distintos niveles de organización; sin embargo, de tal definición derivan conceptualizaciones centradas en el aprovechamiento de la diversidad biológica como parte de un proceso económico y las circunstancias que de él se derivan.

\begin{abstract}
A la gente le interesa la diversidad biológica. Los zoológicos, acuarios, jardines botánicos atraen mucho público. Sin embargo, más allá del conocimiento de unas pocas especies carismáticas, el público no es consciente del papel vital que juega [sic] la diversidad biológica en el suministro de elementos esenciales para nuestra supervivencia y bienestar. (Organización de las Naciones Unidas, s. f.).
\end{abstract}

Esta clase de discursos pueden fomentar reflexiones y prácticas asociadas a una mirada instrumentalista de la naturaleza, de allí que el presente texto inste en alejarse de ese enfoque, buscando una perspectiva que brinde espacio al quehacer pedagógico. Por ello adoptamos el término biodiversidad, que, si bien se considera una contracción de la expresión diversidad biológica, nos posibilita un mayor reconocimiento de su complejidad.

En consonancia con lo propuesto por Martínez (2002), la biodiversidad es el producto de la acción conjunta de procesos ecológicos y evolutivos, como la selección natural, la especiación, la competencia o variabilidad genética de las poblaciones, la cual se estructura desde la acción de tres componentes:
- Diversidad en el aspecto genético. Se entiende desde el papel de la variabilidad genotípica de cada individuo, como medio para los procesos evolutivos y, por ende, de diversificación. Esta es causada por la mutación y la recombinación (Martínez, citado en Soler, 2002).

- Diversidad específica. Se relaciona con el proceso de especiación, al que considera como el principal. Es el resultado de los procesos microevolutivos y macroevolutivos (García, 2005).

- Diversidad de comunidades. En lo que respecta a los elementos productores de diversidad biológica a niveles mayores, cabe destacar las interrelaciones propias de las comunidades, como la competencia y la depredación (Primack, 2000).

Estos conceptos se aceptan tanto desde la biología como desde las disciplinas que componen su campo de saber. A pesar de ello, es preciso preguntarse si esta mirada goza de la misma aceptación en todos los ámbitos académicos y sociales, especialmente el educativo, ya que de acuerdo con Herrera (2016) no existe una única definición de biodiversidad, por lo que su abordaje implica no solo hablar de las diversas formas de lo vivo, sino que también supone reconocer el discurso tras ella, su origen y las dinámicas que implica.

En consonancia, Pérez (2011) identifica la biodiversidad desde una concepción cultural que implica reconocer la interpretación de lo vivo que prepondere a través de conocimientos situados mediante imaginarios sociales. Se infiere que la biodiversidad se ubica como un concepto con múltiples aristas, que incide en lo político, lo biológico y lo cultural tanto como en los aspectos económicos y educativos (Maclaurin y Sterelny, 2008).

El abordaje de la biodiversidad en el marco educativo suele discurrir en el ámbito de la educación ambiental, ya que "[...] es un excelente formato interdisciplinar, para preparar a los ciudadanos del mundo en hacer frente a problemas de la biodiversidad" (Peyton, Campa y Winterstein, 1995, p. 59). De acuerdo con estos autores, las nociones con este enfoque son recurrentes; algunas de ellas incluso se apoyan en elementos como la alfabetización y el diseño de planes curriculares y estrategias para incorporar este tema en el escenario educativo. No obstante, al preguntarse por las prácticas que conducen a la participación de la biodiversidad en el escenario educativo colombiano, se encuentra que estas no tenido un rol establecido durante la última década, aunque sí existen múltiples temarios que la incluyen, especialmente aquellos relacionados con la ecología (Cárdenas, 2015). En consecuencia, desde este concepto es posible reconocer, participary construir reflexiones y análisis en torno a ella. 
Al extender la temática de la biodiversidad a los ejercicios investigativos de los profesores (el cual es uno de los intereses de este trabajo) se busca ampliar su comprensión desde el saber pedagógico, considerando elementos discursivos y procedimentales como un aspecto clave del proceso de enseñanza y como producto de la acción del maestro, susceptible en ese caso de problematizar su saber.

\section{Pensar una estrategia pedagógica}

Comúnmente se piensa que una estrategia es un plan de acción para alcanzar un objetivo a corto plazo (Harrison y John, 2002). A pesar de ello, esta perspectiva no se puede extrapolar directamente al ámbito investigativo, pues se corre el riesgo de equiparar su aplicabilidad a la de un instrumento o una técnica, por lo que se insta a un enfoque que además de presentar el aspecto procedimental involucre una propuesta estructurada que dé cuenta del proceso de construcción y ejecución.

En palabras de Tobón (2004), una estrategia se considera como el modo operante y la forma de proceder, es decir, la definición de un camino que supone una conceptualización relativa al qué, por qué, para qué, cuánto, dónde y cuándo se quiere obtener, asumiendo que lo metodológico no se puede despojar de lo conceptual. Esto permite la teorización de los contenidos de aquello que se pretende conseguir, de la mano con la planeación y los recursos que exige el proceso. Esta última definición contempla dos aspectos fundamentales para tener en cuenta en este estudio: el desarrollo metodológico y el conceptual, los cuales necesitan acercarse a las posibilidades que brinda la práctica pedagógica y la investigación.

En ese orden de ideas, y siguiendo a Cubides y Romero (2010), una estrategia pedagógica se fundamenta en una alternativa para la búsqueda e implementación de nuevos procesos pedagógicos, que faciliten a los estudiantes el aprendizaje de las disciplinas.

De igual manera, Camacho, Flórez, Aguirre, Pasive y Murcia (2012) abordan la estrategia pedagógica desde cuatro referentes: el desarrollo de acciones encaminadas al aprendizaje, la conducción del estudiante a cuestionarse sobre su propio saber, la participación de la lúdica y de herramientas tecnológicas, y finalmente el componente socioafectivo. Entre estas nociones destacan criterios como la vinculación del estudiante con su objetivo de saber y la importancia del factor afectivo; no obstante, es preciso cuestionarse sobre el hecho de facilitar el aprendizaje en una estrategia pedagógica.
En contraste, en este texto se hace un llamado a incluir el proceso de enseñanza como parte de una estrategia pedagógica. Para ello, los autores se apoyan en la propuesta de Westbrook et ál. (2013), según la cual una estrategia pedagógica significa y conlleva las disposiciones de los maestros hacia la enseñanza y el aprendizaje, como la expresión más concreta de su enfoque. Así, permite visibilizar el proceso educativo en la interacción de estas acciones.

Es preciso advertir sobre la existencia de nociones que ponen en sinonimia a las estrategias pedagógicas con las didácticas. De acuerdo con los fundamentos establecidos por De la Torre (2005), una estrategia didáctica se define como una técnica que pretende dinamizar el proceso de aprendizaje y cuyo derrotero se ubica en la constitución de herramientas que desarrollen el espíritu creativo en medio del aprendizaje de los contenidos (Rosales, 2010).

De allí que la presente investigación aborde su propuesta desde una estrategia pedagógica, considerando el saber pedagógico como su medio de enunciación, disertación y problematización. En primer lugar, se encuentra la estructuración y aplicación de pautas teóricas, procedimentales y reflexivas que propicien el desarrollo de saberes provenientes de múltiples ámbitos. Así las cosas, se busca aproximarse a la enseñanza como el objeto de la estrategia y punto de partida en la configuración de los saberes, indagando acerca de los intereses de los estudiantes, las incidencias del contexto de la práctica y el papel del maestro en la investigación.

Se promueve la formulación de estrategias pedagógicas que involucren la indagación de saberes de la mano con la enseñanza (en interacción directa con los actores), para posibilitar la participación de marcos teóricos, propuestas y posiciones en torno a los intereses investigativos y las necesidades contextuales. En este contexto, las estrategias pedagógicas fungen como la materialización del conjunto de ideas, elementos, conceptos y métodos nacidos desde la conceptualización y el análisis del saber pedagógico.

En este orden de ideas, es posible acercarse al modo en que se circunscriben los saberes en la escuela. En el caso que nos ocupa en este estudio -el de biodiversidad y educación no convencional-, lo anterior nos permitirá visibilizar los elementos que caracterizan y contribuyen a estructurar un cuerpo de objetos alrededor de la enseñanza. De allí que esta propuesta de estrategia sea el medio que facilite el acercamiento de los estudiantes a la conceptualización de la biodiversidad. 


\section{Materiales y métodos}

Gracias al entendimiento de los propósitos de la investigación es imperativo posicionarse alrededor de perspectivas epistemológicas y metodológicas en función del reconocimiento de los saberes que fluctúan en el marco del escenario por tratar y su movilización desde la implementación de la estrategia pedagógica. En este sentido, se deben precisar medios que problematicen a quién se dirige y cómo piensan y actúan los sujetos frente a alguna situación, de manera que su comportamiento no es producto de la casualidad sino de complejas subjetividades. Considerado tal aspecto, se adopta un paradigma interpretativo.

De acuerdo con Ramírez (2012), su finalidad se encuentra en problematizar las diferencias existentes entre los fenómenos sociales, fundamentados en la configuración de modelos, conceptos y esquemas a la luz de la experiencia, a fin de reconocer la complejidad y el carácter de los sujetos en relación con su realidad (Schwandt, 2000). Al concebir la enseñanza como una práctica social, como experiencia viva de los sujetos en la construcción de los saberes, es preciso visibilizar y acercarse a las dinámicas que tienen los maestros en formación en el desarrollo de la práctica pedagógica y el modo en que sus propuestas se pueden configurar como un insumo para el reconocimiento de los intereses de los estudiantes frente a la biodiversidad.

Ante el requerimiento de fundamentos que permitan a los licenciados en formación llevar el tema a dimensiones funcionales, sociales y culturales que demandan los contextos, en el proceso de enseñanza y el reconocimiento se ve la conveniencia de hacer una propuesta de corte cualitativo a partir del desarrollo de hipótesis que no busquen la explicación de los sucesos sino las inferencias y los patrones entre los actores, por lo que se requiere una lectura e interpretación de las situaciones concretas, en un enfoque amplio y flexible, no instrumental. Para González Monteagudo (2001), en el enfoque cualitativo se resalta la importancia del contexto en las dinámicas, por lo que no es posible comprender a los sujetos aislados de ellos, lo que hace necesario un análisis de datos de carácter inductivo, que permita la descripción y comprensión de la realidad plural que caracteriza a los actores estudiados.

En relación con las etapas del proyecto se considera como primer momento la incorporación del investigador a la población, a través de su participación en la figura de SSA del Jardín Botánico José Celestino Mutis, escenario donde los estudiantes cuentan con etapas formativas y aplicativas. El objetivo de la experiencia es reconocer elementos relacionados con el territorio y el cuidado de la naturaleza, para después formular e implementar la deno- minada iniciativa ambiental, que consiste en el desarrollo de sesiones con estudiantes de menor grado en relación con temas escogidos por los participantes (JBB, 2014). Se contó con un grupo aproximado de 45 estudiantes, compuesto en su mayoría por varones entre los grados noveno y undécimo, con edades comprendidas entre los $14 \mathrm{y}$ los 18 años.

El papel del investigador en este caso es el de acompañar las sesiones formativas como tutor en el momento de la implementación. La finalidad de esta etapa es la caracterización de los aspectos educativos del lugar, en el contexto de las distintas dinámicas que tienen lugar en este escenario y del modo en que se tejen situaciones problema en relación con la biodiversidad. Así pues, el principal medio de recolección de datos es la observación directa y su registro en el diario de campo.

Posteriormente, tiene lugar la identificación y el análisis de los elementos que puedan dar cuenta del modo en que la biodiversidad se presenta a los ojos de los estudiantes: sus opiniones, perspectivas y cocimientos al respecto. Este objetivo se alcanza gracias al análisis de la información proveniente de la aplicación de herramientas de recolección (como encuestas, diarios de campo, análisis documental y registro de observaciones), y del desarrollo de sesiones formativas en liderazgo por parte del investigador.

Una vez comprendida la intención investigativa y las posibilidades que brinda el escenario, se insta a la construcción de una estrategia pedagógica, con el fin de que los sujetos reconozcan algunos elementos relacionados con la biodiversidad. En esta última instancia tiene lugar la delimitación de los conceptos, la indagación teórica y la aproximación a los saberes de los actores, de modo que es posible llevar la temática al contexto educativo para aportar a la construcción de los conocimientos y encauzar la enseñanza de temas que involucran la biodiversidad.

En lo que respecta a la estructuración de la estrategia, se pone en escena una serie de ideas, propuestas, actividades y dinámicas que configuran el cuerpo de las sesiones; en cada una se plantean elementos conceptuales que tienen que ver con la biodiversidad. En la tabla 1 se presenta una matriz detallada de las etapas expuestas y se describen las actividades, técnicas e instrumentos que tienen lugar. 
Tabla 1. Etapas en la estructuración de la estrategia

\begin{tabular}{|c|c|c|c|}
\hline Sesión & $\begin{array}{c}\text { Temas centra- } \\
\text { les }\end{array}$ & Objetivo & Descripción \\
\hline $\begin{array}{l}\text { Cómo abordar la } \\
\text { biodiversidad }\end{array}$ & $\begin{array}{l}\text { Diversidad } \\
\text { específica. } \\
\text { Clasificación } \\
\text { de organismos } \\
\text { vegetales. }\end{array}$ & $\begin{array}{l}\text { Identificar aspectos } \\
\text { relacionados con } \\
\text { la biodiversidad } \\
\text { específica y la } \\
\text { clasificación de los } \\
\text { organismos. }\end{array}$ & $\begin{array}{l}\text { Se solicita a los estudiantes que delimiten una zona del suelo a través de } \\
\text { una parcela, en la cual deben caracterizar la artropofauna, la flora y, en los } \\
\text { casos presentes, macrohongos, ilustrando y detallando las características } \\
\text { que observan. En el caso de las plantas, deben clasificarlas de acuerdo con } \\
\text { la subdivisión y, de ser posible, la clase taxonómica en que se encuentren } \\
\text { (Monocotiledónea y Dicotiledónea) y aproximar el número de ejemplares } \\
\text { que hallen. Finalmente, de acuerdo al organismo caracterizado se realiza } \\
\text { una tabla de composición en la que se presenten la cantidad de organismos } \\
\text { encontrados y los morfotipos evidenciados. } \\
\text { En un segundo espacio se realiza la retroalimentación del proceso y se } \\
\text { presentan las disertaciones respectivas utilizando como apoyo visual un } \\
\text { mapa filogenético que caracteriza la diversidad de grupos de organismos e } \\
\text { introduce a los estudiantes a los sistemas de clasificación. }\end{array}$ \\
\hline $\begin{array}{l}\text { La biodiversidad en } \\
\text { los ecosistemas }\end{array}$ & $\begin{array}{l}\text { Diversidad } \\
\text { ecosistémica. } \\
\text { Interacciones en } \\
\text { organismos. } \\
\text { Factores físicosy } \\
\text { geográficos. }\end{array}$ & $\begin{array}{l}\text { Identificar aspectos } \\
\text { relacionados con } \\
\text { la biodiversidad } \\
\text { ecosistémica. }\end{array}$ & $\begin{array}{l}\text { En grupos, los estudiantes deben movilizarse a las zonas propuestas en el } \\
\text { JBB (Páramo, Subxerofítico, Humedal, Bosque de helechos) y caracterizar } \\
\text { el ecosistema, resaltando aspectos como las formas de las plantas y la } \\
\text { presencia de factores abióticos, y regresar al punto de encuentro para } \\
\text { socializar con sus compañeros lo encontrado. } \\
\text { Se estructuran las siguientes preguntas problema que funcionan como un } \\
\text { marco para introducir a los estudiantes al ejercicio propuesto: } \\
\text { 1. ¿Cómo es la vegetación del lugar? } \\
\text { 2. ¿El entorno es húmedo o seco? } \\
\text { 3. ¿Encontramos agua en ese lugar? } \\
\text { 4. ¿Ves alguna interacción en ese lugar? } \\
\text { 5. ¿Hay mayor cantidad de Monocotiledóneas o Dicotiledóneas? } \\
\text { Durante la socialización se hacen las consideraciones necesarias, y a partir } \\
\text { de lo presentado por los estudiantes se habla de elementos relacionados } \\
\text { con las interacciones entre los organismos y entre los ecosistemas. Además, } \\
\text { se proponen aspectos que vinculan factores geofísicos que producen las } \\
\text { características en los ecosistemas. }\end{array}$ \\
\hline $\begin{array}{l}\text { La diversidad y sus } \\
\text { dinámicas }\end{array}$ & $\begin{array}{l}\text { Dinámica de } \\
\text { poblaciones. }\end{array}$ & $\begin{array}{l}\text { Identificar aspectos } \\
\text { relacionados } \\
\text { con los cambios } \\
\text { poblacionales y la } \\
\text { biodiversidad. }\end{array}$ & $\begin{array}{l}\text { Siguiendo la dinámica de encuentros anteriores, los estudiantes se separan } \\
\text { en equipos de trabajo. A cada uno de ellos se le asignan cantidades y } \\
\text { variedades de dulces de colores, con el fin de cuantificar la totalidad de } \\
\text { las unidades e interpretarlas como individuos de una población. Luego se } \\
\text { extraen aleatoriamente unidades del grupo en analogía de las fluctuaciones } \\
\text { en las poblaciones y se evalúan las inferencias de los participantes al } \\
\text { respecto. } \\
\text { Se solicita la siguiente información: } \\
\text { 1. ¿Qué cantidad de dulces hay de cada color? } \\
\text { 2. ¿Luego de los cambios (extracción de unidades) cuáles características } \\
\text { tiene más posibilidad de sobrevivir? } \\
\text { 3. ¿Qué función pueden tener los dulces de color verde? } \\
\text { 4. ¿Qué número de dulces de cada color hubo luego de la extracción? } \\
\text { 5. ¿En } 10 \text { generaciones que color será más abundante? }\end{array}$ \\
\hline $\begin{array}{l}\text { Biodiversidad y la } \\
\text { Tierra }\end{array}$ & Biogeografía. & $\begin{array}{l}\text { Distinguir aspectos } \\
\text { relacionados } \\
\text { con la influencia } \\
\text { geográfica y } \\
\text { geológica en la } \\
\text { biodiversidad. }\end{array}$ & $\begin{array}{l}\text { Al inicio del encuentro se hace una conceptualización de los aspectos } \\
\text { biogeográficos que tienen lugar en la biodiversidad. Se presentan los temas } \\
\text { "Regiones biogeográficas", "Climas de la tierra" " "Movimientos tectónicos", } \\
\text { con la finalidad de elaborar un mapa de Colombia y uno de Suramérica } \\
\text { y relacionar los elementos conocidos por los estudiantes - además de } \\
\text { los presentados en la sesión- para con ello visibilizar las diferencias } \\
\text { geográficas. } \\
\text { En los mapas cada estudiante representa por medio de dibujos y/o textos } \\
\text { los elementos geográficos que logre diferenciar. Además, el investigador } \\
\text { los anima a interrogarse acerca de la incidencia de tales factores en la } \\
\text { biodiversidad. }\end{array}$ \\
\hline
\end{tabular}




\section{Resultados y discusión}

A continuación se presentan los aspectos generales encontrados a partir del análisis de las sesiones desarrolladas durante la implementación de la estrategia. Se destacan algunos elementos claves que se amplían más adelante.

\section{Primera sesión: cómo abordar la biodiversidad}

Un elemento recurrente a lo largo del acercamiento con la población consistió en la conceptualización de la biodiversidad con base en las características que diferencian a los organismos, por lo que esta propuesta brindó un elemento conocido por los estudiantes para vincular a partir de este, otros marcos teóricos que enriquecen la pregunta acerca de la biodiversidad. En un primer momento, se consideró la biodiversidad específica como el tema central de la sesión; a partir de este emergieron conceptos como riqueza, abundancia y clasificaciones taxonómicas.

En relación con el desarrollo de la sesión se destacó el interés de los estudiantes al entrar en contacto con los organismos. Por consiguiente, la observación de sus características más evidentes resultó en el reconocimiento de varios grupos de animales y plantas, que además de ser relacionados con los conocimientos previos de los estudiantes dieron lugar a emociones como el afecto y la familiaridad. Esto sugiere que el modo en que los estudiantes contemplan la biodiversidad parte de la apariencia física de los organismos y de sus experiencias previas con ellos.

En lo concerniente a los sistemas de clasificación, se encuentra que los estudiantes desconocen la clasificación en los tres dominios (Woese, Kandler y Wheelis, 1990) y están más familiarizados con la clasificación en cinco reinos (Whittaker, 1969). Finalmente, se destaca el interés demostrado por los estudiantes frente a grupos de animales singulares (Tardígrada, Priapúlida, Platemytes, Rotífera), en la mayoría de los casos desconocidos.

\section{Segunda sesión: la biodiversidad en los ecosistemas}

Con el fin de permitir a los estudiantes reconocer las diferencias entre los ecosistemas se destacan las posibilidades que brinda el JBB en cuanto espacio compuesto por numerosas zonas vegetales con una gran variedad de organismos. De ahí que sea necesario adoptar metodologías que permitan aprovechar tal situación, de manera que el contacto directo con estos elementos constituya un insumo para el desarrollo de prácticas educativas asociadas a la biodiversidad.
Una de las finalidades del ejercicio fue concebir los ecosistemas como un entramado de múltiples interacciones. Al respecto, la mayoría de los estudiantes se refirió a las relaciones de tipo planta-animal basándose en el acercamiento que tuvieron y la posibilidad de observar tal situación, lo que lleva a concluir que los estudiantes reconocen las relaciones entre los organismos que son más evidentes o aquellos con los que han tenido referencia previamente. Los estudiantes no tuvieron ninguna dificultad en la caracterización de los ecosistemas, especialmente en lo que tiene que ver con el reconocimiento de aspectos fisicoquímicos y ambientales como temperatura, humedad, presencia de agua, etcétera.

\section{Tercera sesión: La diversidad y sus dinámicas.}

Esta jornada se centró en temas evolutivos, en consideración de temáticas como deriva genética y efecto fundador. Se evidenciaron falencias conceptuales en los estudiantes en temas como el tiempo profundo, los cambios geológicos y las dinámicas de poblaciones, lo que implica tener en cuenta un acercamiento teórico a la ecología de poblaciones y la genética para posteriores ejercicios. A pesar de ello, lograron identificar la existencia de cambios en los organismos y variaciones en sus poblaciones a lo largo del tiempo.

\section{Cuarta sesión: biodiversidad y la tierra}

Finalmente, en esta sesión se dio el acercamiento a las relaciones existentes entre las condiciones geográficas y geológicas y la biodiversidad. Se puso de presente la vinculación de estos aspectos con las actividades económicas predominantes; asimismo, se señaló una tendencia a destacar las divisiones políticas sobre las condiciones naturales o biológicas. Con respecto al análisis de los productos realizados por los estudiantes, se destaca la similitud entre ellos, en especial en la categorización propuesta de las condiciones climáticas de distintas zonas del país, el reconocimiento de animales y vegetales asociados a cada sector y las condiciones físicas que inciden en la composición de la biodiversidad.

Si se tiene en cuenta que este texto se concibió con un enfoque interpretativo, es posible que se caiga en miradas subjetivas e inherentes al modo de ver del investigador. Por esta razón, es necesario establecer diálogos con los actores con los que se desarrolló la estrategia.

En relación con la significación de la biodiversidad por parte de los estudiantes emerge una interpretación generalizada hacia la "variedad de animales y plantas" ligadas a su existencia en el ambiente y el ecosistema. Este último 
elemento hace explícita una nueva vinculación de la biodiversidad con la óptica de los estudiantes, lo que sin duda representa una posibilidad para desarrollar en próximos ejercicios. Vale destacar posturas que incluyen la categorización de la biodiversidad de acuerdo con niveles de organización (genético, específico y ecosistémico), situación que se podría explicar por el ejercicio realizado por el investigador a lo largo del desarrollo de la estrategia.

Al indagar por los elementos conceptuales que se tuvieron en cuenta en la estrategia, desde la perspectiva de los estudiantes, se mencionan múltiples aspectos vinculados a las actividades y a las temáticas desarrolladas. La mayoría de los estudiantes destacan elementos como especies representativas de distintas zonas del JBB, clasificación de organismos y diversidad genética; así se pone de presente el papel de los escenarios de educación no convencional y la pertinencia de la estrategia.

Ahora bien, los estudiantes manifiestan la necesidad de incorporar dentro de las sesiones formativas del SSA temáticas relacionadas con los temas que desarrollan en sus iniciativas. Si bien esta situación puede reflejar el desconocimiento de dinámicas propias del proceso, se puede tener en cuenta en los temas más recurrentes en el desarrollo de las iniciativas.

Durante una primera aproximación a la población escolar y en la mayor parte de esta investigación se encontró que en algunos casos la importancia atribuida a la biodiversidad estaba influenciada por nociones instrumentalistas en aspectos como obtención de recursos naturales y proyectos de interés agrícola, lo que desde luego conduce a una serie de problematizaciones en relación con el origen de estas implicaciones y las prácticas que pueden suscitar.

Por el contrario, en la pregunta sobre los saberes asociados al conocimiento de la biodiversidad resaltan enfoques vinculados a la variedad de las formas de lo vivo, como el origen de las especies, su clasificación, la diversidad de animales y plantas, distintos ecosistemas, claves taxonómicas, la naturaleza, necesidad de cuidado y el comportamiento. Este panorama dista de los resultados obtenidos por Menzel y Bögeholz (2009), quienes al indagar en una población escolar por la biodiversidad relatan perspectivas ligadas principalmente a los servicios ecosistémicos y la educación ambiental.

Vale la pena considerar como el aspecto más representativo de la investigación la diferenciación de la importancia de la biodiversidad frente a su conocimiento, lo que lleva a contrastar perspectivas instrumentalistas, activistas y ecologistas frente a los discursos de las ciencias naturales y biológicas. Esta situación se observa en las formas en que los estudiantes utilizaron el concepto de especie: en un primer momento aluden a la diversidad de especies y su cuidado, pero al explorar más a fondo se encuentran opiniones ambiguas; incluso llegan a proponer el concepto como sinónimo de alelo o raza. Si bien esto revela influencia del discurso biológico en las miradas de los estudiantes, también denota la polisemia y la discusión epistemológica alrededor de la biodiversidad.

Michán (2010) menciona que la diferenciación entre los organismos a nivel macroscópico resulta ser el elemento fundamental en los ejercicios asociados a los saberes de la biodiversidad, lo cual concuerda con las observaciones presentadas, evidenciadas especialmente en las caracterizaciones propuestas por los estudiantes a lo largo de las sesiones. Durante el desarrollo, se hicieron planteamientos como la necesidad de conservación de la biota, la apreciación de los animales y las plantas, la posibilidad de relacionarse directamente con la biodiversidad, el cuidado de los ecosistemas y la enseñanza acerca de los insectos a la comunidad. Asimismo, el papel de la biodiversidad no se encontró únicamente en la adquisición de conceptos sino en la posibilidad de proponer unos nuevos, lo que se presume como punto de partida para la configuración de nuevas miradas sobre la biodiversidad, y su potencial para incluirla en el ejercicio educativo de los maestros de biología.

Se evidenció una estrecha relación entre la formación académica y el contexto en que se desenvuelven los sujetos en correspondencia a la significación de la biodiversidad. Las múltiples nociones con respecto al tema pueden ser visibles desde el acercamiento previo a este o su reconocimiento. Tal situación da cuenta de cómo los distintos discursos alrededor de la biodiversidad tienen influencia en su interpretación.

Cárdenas (2015) afirma que en el currículo colombiano no se abordó la biodiversidad en la primera década del presente milenio, y propone su inclusión en otros temarios con una estructura posicionada. Desde el desarrollo de esta fue posible localizar la biodiversidad en los discursos de la educación ambiental, la ecología, la zoología, la botánica, la micología, la microbiología y la genética. De este modo, el lugar de la biodiversidad encontrado desde esta investigación parte de la interacción de múltiples instancias de las ciencias biológicas y educativas, y se acerca a la validación de las premisas propuestas. Además, lleva a preguntarse por la posibilidad de análisis frente al modo en que influye la biodiversidad en los ámbitos en los que se identificó.

Un aspecto recurrente durante el ejercicio fue el de las relaciones entre biodiversidad y educación ambiental. Si bien esta dinámica escapa a los intereses específicos 
de la investigación, emergió en el entendimiento de su amplia generalización. Esto se demostró en la vinculación de los estudiantes a los discursos de la educación ambiental, definida como una apuesta para el cuidado y preservación del entorno. Sin duda, esta situación implica explorar los marcos teóricos de la educación ambiental como parte del análisis de los saberes de los estudiantes acerca de la biodiversidad.

De acuerdo con los hallazgos, la biodiversidad se expresa como la fuente de beneficios y la prolongada lucha por protegerla, por lo que su importancia dista de ser contemplada desde su valor implícito, apreciativo o afectivo para encontrar apogeo en una tónica instrumental, mercantilista o ambientalista. Esto, sin duda, supone nuevos retos para los maestros de ciencias naturales, biología, o los educadores ambientales. Aun cuando las lógicas de la contemporaneidad perfilan las concepciones frente a la importancia de la biodiversidad, es admisible una apuesta contextualizada, alternativa y diferenciada al respecto, que encuentre cabida en el modo en que los sujetos contemplan la variedad de lo vivo.

Incluso en las perspectivas de los estudiantes con relación al desarrollo y la pertinencia del SSA se destacan nociones como la preservación del ambiente, la búsqueda de soluciones a problemáticas ambientales, la necesidad de cuidado del ambiente y las relaciones existentes entre naturaleza y ambiente, por lo que no es difícil inferir la inclinación de los estudiantes hacia el discurso de la educación ambiental, elemento recurrente a lo largo de la práctica.

Es posible definir el lugar donde se desarrolló la investigación como un espacio educativo caracterizado por un componente curricular flexible, abierto a la participación del saber pedagógico. Esto explica la categoría "Escenarios de educación no convencional" propuesta en el título de este texto, que se utilizó con el fin de llamar a la incorporación de metodologías, análisis y reflexiones respecto al papel del concepto de biodiversidad en estudiantes fuera de los espacios tradicionales de enseñanza.

Este ejercicio implicó el reconocimiento de los escenarios de educación no convencional como lugares para una interacción constante entre teoría y práctica. El desarrollo de la práctica pedagógica se vio influenciado por las condiciones que los actores y el contexto dinamizan, por lo que el conocimiento producido en estas instancias se singulariza en los saberes de tal interacción, y constituye, en cualquier caso, un elemento plausible en los análisis de los maestros.

\section{Reflexiones finales}

Recoger el acontecer de la práctica pedagógica implica ponerse en el lugar del futuro maestro de biología. Esto constituye un marco de nuevas preguntas y múltiples retos que llevan a pensar el ejercicio desarrollado como una experiencia personal y colectiva que permite acercarse a los saberes que existen en el escenario escolar, entendiendo la multiplicidad de ámbitos relacionados con la labor pedagógica. De ahí la importancia de tener en cuenta el carácter crítico, reflexivo, propositivo, analítico y sistemático inherente a la enseñanza.

Así las cosas, al analizar los saberes de la biodiversidad en los escenarios de educación no convencional se encuentran elementos conceptuales y metodológicos que permiten dar cuenta de sus posibilidades educativas. De allí que una estrategia pedagógica dé cuenta de dichas posibilidades, pero también ponga en evidencia los múltiples discursos, concepciones y prácticas alrededor de la biodiversidad.

\section{En relación con la biodiversidad}

La construcción del presente texto no es exclusiva del concepto biodiversidad, por el contrario se desarrolla desde el diálogo de múltiples posturas orientadas a reconocer los principales elementos que la representan. Esto lleva a considerar el término de manera polisémica y centrada en la variabilidad de las formas de vida. Se diferencia del concepto de diversidad biológica con el fin de desviar la atención de la connotación mercantilista y utilitarista que caracteriza a este último.

Al adentrarse en el escenario, en un primer momento surge la sospecha por una mirada instrumentalizada de la biodiversidad. Esto sugiere la participación de una serie de conceptos de la biodiversidad en la escuela y su interacción con la cotidianidad de los sujetos, por lo que vale la pena preguntarse por la posibilidad de intervención de los maestros y el desarrollo de ejercicios pedagógicos.

Durante este último proceso surge el cuestionamiento acerca del lugar de la biodiversidad en la educación no convencional. Se indaga sobre las posibilidades para su enseñanza, centradas en el desarrollo y análisis de cuatro series de actividades que abarcan los siguientes niveles de la biodiversidad: genético, específico, ecosistémico, geológico y geográfico. Desde esta lógica se puede afirmar la coexistencia de múltiples discursos y prácticas que trascienden la perspectiva instrumental mencionada, y se establecen las voces de los sujetos en relación con el concepto de biodiversidad, diferenciando su importancia, su campo de conocimiento y los medios con los que se vincula al hombre. 
En síntesis, la polisemia del término se ve reflejada en los saberes que atañen a los sujetos tratados, quienes logran abordar sus distintas aristas, pero también se constituye en una evidencia del papel de los maestros de biología.

\section{La educación en escenarios no convencionales}

La falta de antecedentes con respecto a estos escenarios sirve en este ejercicio como la excusa para identificar su estructura, dinámicas, potencialidades, carencias y retos. Con ello se espera entender los escenarios de educación no convencionales como la confluencia de elementos de la educación no formal e informal, que toca las puertas de públicos diversos. Estos contextos tienen características muy marcadas, de acuerdo con la institución desde donde se analiza la práctica pedagógica. Por lo que se insta a cuestionarse el modo en que sus diferencias dan lugar a prácticas diversas y particulares.

En el caso puntual del JBB se destacan intereses educativos marcados en los objetivos institucionales propuestos, situación coherente con la lógica de una institución centrada en la búsqueda de protección de la naturaleza, la construcción y apropiación del conocimiento. Se contempla en ese caso el SSA como ruta de posibilidades frente al reconocimiento de la naturaleza, pero también al entendimiento del modo en que se produce ese recogimiento. Se propone entonces vincular la pedagogía y la didáctica como medio de problematización, proposición y reflexión al respecto.

Desde esta óptica, la práctica pedagógica logra poner en circulación, además de respuestas a una situación problema, un profundo marco de reflexión y análisis del lugar de la pedagogía y de los maestros. Así, se establece desde allí el propósito esencial de la educación y el ejercicio de la enseñanza.

Para finalizar, se presentan la práctica y la investigación pedagógicas como un espacio abierto, configurado por los tránsitos y encuentros con los sujetos, el contexto y los contenidos. En consecuencia, es posible situar las voces de los maestros en búsqueda de la recuperación de la enseñanza, su sistematización y análisis como elementos exclusivos con los cuales se pueden contemplar el aprendizaje y la enseñanza.

\section{Referencias}

Amaya, R. (2007). La investigación en la práctica educativa: guía metodológica de investigación para el diagnóstico y evaluación en los centros docentes (vol. 5). Ministerio de Educación y Ciencia de España.

Calvo, G., Camargo, M. y Pineda, C. (2012). ¿Investigación educativa o investigación pedagógica? El caso de la investigación en el Distrito Capital. Magis. Revista Internacional de Investigación en Educación, 1(1), 163-174.

Camacho, T., Flórez, M., Gaibao, D., Aguirre, M., Pasive, Y. y Murcia, G. (2012). Estrategias pedagógicas en el ámbito educativo. https://docplayer. es/13194924-Estrategias-pedagogicas-en-el-ambito-educativo.html.

Cárdenas, J. (2015) Aproximación a la conformación discursiva de la biodiversidad en la escuela colombiana (2000-2010) (tesis de pregrado). Universidad Pedagógica Nacional, Bogotá, Colombia.

Cubides, E. y Romero, Y. (2010). El club de ciencias: ¿Por qué constituirlo como una estrategia pedagógica? Bio-Grafía, 3(5), 162-169. https://doi.org/10.17227/20271034. vol.3num.5bio-grafia162.169

García Azkonobieta, T. E. (2005). Evolución, desarrollo y (auto)organización. Un estudio sobre los principios filosóficos de la evo-devo. [Tesis de doctorado, Universidad del País Vasco].

González Monteagudo, J. (2001). El paradigma interpretativo en la investigación social y educativa: nuevas respuestas para viejos interrogantes. Cuestiones pedagógicas, 15, 227-246.

Harrison, J. S. y John, C. H. S. (2002). Fundamentos de la dirección estratégica. Madrid, España: Paraninfo.

Herrera, L. (2016). Aportaciones en torno a la biodiversidad en el escenario escolar y de la educación ambiental: una mirada reflexiva al saber pedagógico. I Congreso Nacional de Educación Ambiental para la Sustentabilidad. México: Tuxtla Gutiérrez.

Jardín Botánico José Celestino Mutis JBB (2014). Servicio social ambiental Jardín Botánico José Celestino Mutis [Archivo de video]. https://www.youtube. com/watch?v=LJyaoz93vus.

De la Torre Zermeño, F. (2005). 12 lecciones de pedagogía, educación y didáctica. México: Alfaomega.

Maclaurin, J. y Sterelny, K. (2008). What is Biodiversity? The University of Chicago Press. 
Martínez, J. G. (2002). La evolución y la conservación de la biodiversidad. En M. Soler Cruz (coord.), Evolución: la base de la biología (pp. 407-416). España: Proyecto Sur.

Menzel, S. y Bögeholz, S. (2009). The Loss of Biodiversity as a Challenge for Sustainable Development: How do Pupils in Chile and Germany Perceive Resource Dilemmas? Research in Science Education, 39(4), 429-447.

Michán, L. (2010). Análisis bibliométrico de la producción sistemática en América Latina. http://www.scielo. org.co/pdf/abc/v16n2/v16n2a3.pdf.

Organización de las Naciones Unidas. (1992). Convenio sobre la diversidad biológica. Texto final del Convenio sobre la Diversidad Biológica. Cumbre para la Tierra. Conferencia de las Naciones Unidas sobre el Medio Ambiente y el Desarrollo. Río de Janeiro, 3 al 14 de junio. https://www.cbd.int/doc/legal/ cbd-es.pdf

Organización de las Naciones Unidas. (s. f.). Convenio sobre la Diversidad Biológica-CDB 2011-2020. Decenio de las Naciones Unidas sobre la Biodiversidad. https://www.cbd.int/undb/media/factsheets/undb-factsheets-es-web.pdf.

Pérez, M. R. (2011). Concepciones de biodiversidad: una mirada desde la diversidad cultural. Magis. Revista Internacional de Investigación en Educación, 6(12), 133-151.

Peyton, B., Campa, H. W. y Winterstein, S. R. (1995). Biological Diversity for Secondary Education: Environmental Education Module. The United Nations Educational, Scientific and Cultural Organization. https://unesdoc.unesco.org/in/rest/annotationSVC/DownloadWatermarkedAttachment/ attach_import_d3e0c0dd-c64e-4a0d-88e0-e992a48f18a1?_=111306engo.pdf

Primack, R. (2000). A Primer of Conservation Biology (2. ${ }^{\text {a ed.). }}$ Sunderland, Mass.: Sinauer Associates.

Ramírez, I. (2012). Los diferentes paradigmas de investigación y su incidencia sobre los diferentes modelos de investigación didáctica. https://bit.ly/2VnECHk

Rosales, J. (2010). Estrategias didácticas. Ponencia presentada durante el Coloquio de red del nivel, Universidad de Guadalajara. http://dcb.fi-c.unam. $\mathrm{mx} /$ Eventos/Foro4/Memorias/Ponencia_17.pdf.

Schwandt, T. A. (2000). Three Epistemological Stances for Qualitative Inquiry. Handbook of Qualitative Research, 2(2), 189-213.
Soler, M. (2002). Evolución: la base de la biología. España: Proyecto Sur.

Tobón, R. (2004). Estrategias comunicativas en la educación: hacia un modelo semiótico-pedagógico. Medellín: Universidad de Antioquia.

Trujillo, L. y Valbuena, E. (2015). ¿Educación no convencional, no formal o informal? Emergencia en el marco de investigación con tres licenciados en biología. Bio-Grafía, edición extraordinaria, 1-11.

Universidad Pedagógica Nacional-Consejo Académico. (2013). Acuerdo 016. Por el cual se expide el reglamento de práctica pedagógica del Proyecto Curricular de la Licenciatura en Biología. Bogotá, Colombia.

Westbrook, J., Durrani, N., Brown, R., Orr, D., Pryor, J., Boddy, J. y Salvi, F. (2013). Pedagogy, curriculum, teaching practices and teacher education in developing countries. Education Rigorous Literature Review. Brighton, uk: Center for International Education, University of Sussex.

Whittaker, R. H. (1969). New Concepts of Kingdoms of Organisms. Science, 163(3863), 150-160.

Woese, C. R., Kandler, O., y Wheelis, M. L. (1990). Towards a Natural System of Organisms: Proposal for the Domains Archaea, Bacteria, and Eucarya. Proceedings of the National Academy of Sciences, 87(12), 4576-4579.

Zuluaga, O. L. (1999). Pedagogía e historia. Bogotá: Ediciones Foro Nacional por Colombia. 